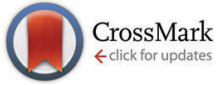

Cite this: Phys. Chem. Chem. Phys., 2015, 17, 6340

Received 4th December 2014, Accepted 26th January 2015

DOI: $10.1039 / c 4 c p 05641 \mathrm{~h}$

www.rsc.org/pccp

\section{Optical discrimination of racemic from achiral solutions $\dagger$}

\author{
Andreas Steinbacher, ${ }^{a}$ Patrick Nuernberger ${ }^{\mathrm{b}}$ and Tobias Brixner $^{\star^{a}}$
}

\begin{abstract}
The use of femtosecond laser pulses to selectively trigger an asymmetric photoreaction is demonstrated in this study. Depending on the handedness of the laser polarization, more $R$ - or $S$-enantiomers of a racemate are gradually destroyed, leading to the generation of enantiomeric excess. The kinetic evolution of this excess is detected with a sensitive polarimeter. Hence, discrimination between racemic and achiral solutions and selective preparation of an enantiomeric excess within seconds is achievable. Furthermore, this technique opens the possibility for quantum control with chiral substances if the laser pulses which trigger the photoreaction are adjusted by pulse-shaping techniques.
\end{abstract}

\section{Introduction}

The concepts of symmetry and symmetry breaking play central roles in fundamental theories of physics, and symmetry properties are relevant for many applications across all disciplines of science and engineering. It is thus of general interest to determine whether a given object of study obeys certain symmetries or not. Chirality is an intrinsic structural property arising from symmetry considerations: a chiral object cannot be superimposed with its mirror image. In the macroscopic domain, optically discriminating chiral from achiral objects is usually straightforward, as the spatial conformation can be deduced directly. For microscopic objects, such as molecules, indirect spectroscopic methods are often employed using circular dichroism (CD) or optical activity (OA). ${ }^{1}$ In both techniques, one exploits the chiral properties of light interacting differently with a molecule and its mirror image, i.e., with enantiomers. These approaches rely on a difference in absorption of left versus right circularly polarized light (CD) or a change in the polarization direction of transmitted linearly polarized light (OA), respectively. ${ }^{2}$ In emission spectroscopy, the chirality of excited molecules is expressed in circularly polarized luminescence. ${ }^{3}$

These effects are commonly exploited to identify the chirality of molecules in solution by optical means. Reaching measurable $\mathrm{CD}$ or OA signals may typically require long optical path lengths, high concentrations, and long integration times. Hence, only few ultrafast studies utilizing CD or OA could be established. ${ }^{4-17}$

\footnotetext{
${ }^{a}$ Institut für Physikalische und Theoretische Chemie, Universität Würzburg, Am Hubland, 97074 Würzburg, Germany. E-mail: brixner@phys-chemie.uni-wuerzburg.de; Fax: +49-931-31-86332; Tel: +49-931-31-86330

${ }^{b}$ Physikalische Chemie II, Ruhr-Universität Bochum, 44780 Bochum, Germany. E-mail: patrick.nuernberger@rub.de; Tel: +49-234-32-29946

$\dagger$ Electronic supplementary information (ESI) available. See DOI: 10.1039/ c4cp05641h
}

Approaches for determining the chirality of a sample in the gas phase with optical techniques were reported recently, detecting the angular distribution of photoelectrons ${ }^{18,19}$ or microwave emission. ${ }^{20}$ By Coulomb explosion imaging ${ }^{21}$ one can even measure the absolute configuration directly.

Here we address a question beyond the established optical procedures in discriminating chiral enantiomers from each other or chiral from achiral samples: can one optically distinguish (1) an ensemble in which all individual objects are achiral from (2) an ensemble in which the individual objects are chiral but exist as an equal mixture of enantiomers, i.e., a racemate? This question is directly connected with the desire for optical enantio-separation, or chiral purification, which recently has drawn some attention. ${ }^{22-24}$ Common nonoptical methods for such discrimination are chirality-sensitive $\mathrm{NMR}^{25}$ or the spatial separation of a racemate into its enantiomer components via, e.g., chiral chromatography, crystallization, or a combination of both. ${ }^{26,27}$ Typically, those are very demanding, costly and/or time-consuming. Thus, we address the question if it is however possible to develop an all-optical discrimination (i.e., being able to distinguish by optical means only) without prior spatial separation. Straightforward optical detection on an unseparated ensemble does not work because $\mathrm{CD}$ or OA of individual chiral molecules in the racemate average out. For an identification via tiny deviations in the band positions predicted due to parity violation, see ref. 28 and 29. Thus, both a racemic and an achiral solution show zero CD and zero OA.

The main concept of the present work is to use accumulative femtosecond ( $\mathrm{fs}$ ) spectroscopy ${ }^{30,31}$ for creating an enantiomeric excess (ee) from the originally racemic solution and to rapidly detect the resulting $\mathrm{OA}$ with an extremely sensitive polarimeter. ${ }^{32}$

In the literature, several optical routes are known for generating ee. ${ }^{33}$ Optical ee generation has been demonstrated almost a century ago using long-term illumination with incoherent 
circularly polarized light ${ }^{34-36}$ leading to asymmetric photodecomposition. Exploiting the CD effect, one enantiomer absorbs light of one handedness stronger than the other and thus preferentially photodissociates if such a channel is available, causing an ee of the other enantiomer. We transfer this idea to state-of-the-art fs laser spectroscopy allowing us (1) to generate and detect ee within seconds rather than days of illumination, (2) to follow the dynamical evolution of the asymmetric decomposition in quasi real-time, i.e., with a $100 \mathrm{~Hz}$ sampling rate, and (3) to enable future scenarios of chirality control with shaped fs laser pulses. For the latter, various schemes have been suggested using circularly ${ }^{37,38}$ or combinations of linearly polarized laser fields ${ }^{39-45}$ but no successful experiment has been reported yet in solution.

\section{Experimental setup}

The setup which is capable of optically discriminating racemic from achiral solutions is depicted in Fig. 1a. We employ a sensitive polarimeter to detect the pump-pulse-sequence-induced opticalrotation (OR) change $\Delta \alpha$ in a capillary containing the sample. Details on the mode of operation and data recording can be found in ref. 32. The main components of the polarimeter are a continuous-wave (CW) $405 \mathrm{~nm}$ laser diode, a linear polarizer, an electro-optic modulator (EOM), a Berek-type variable wave plate, and another linear polarizer working as analyzer. By driving the EOM with a saw-tooth voltage with a magnitude of twice the halfwave voltage of the EOM, the resulting polarizations constitute

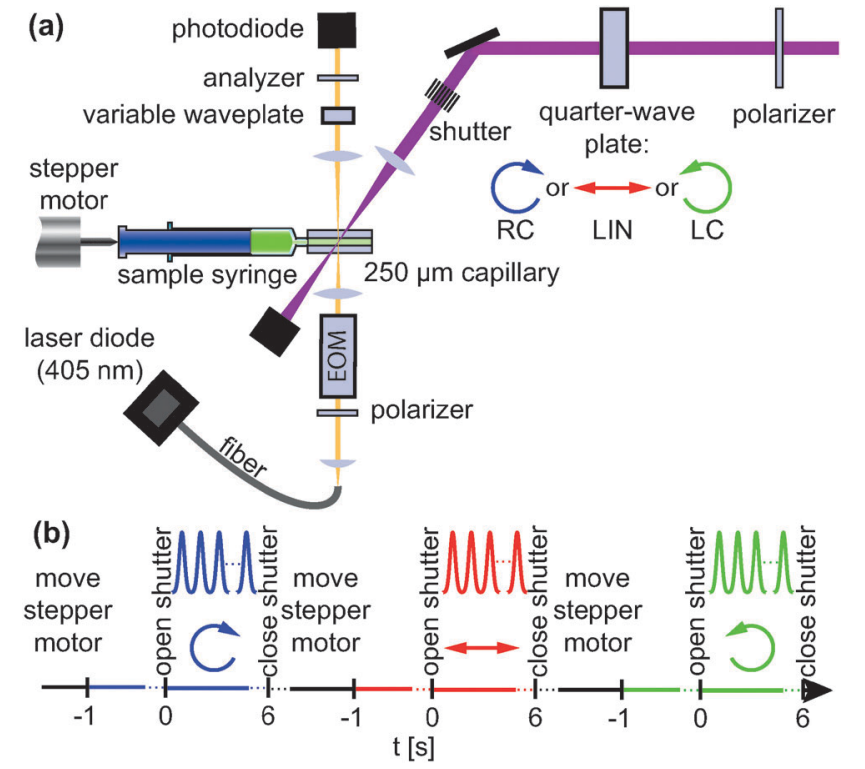

Fig. 1 (a) Scheme of the experimental apparatus. Pump (purple) and probe (orange) beams are spatially overlapped in a sample capillary to record OR values before and during illumination with fs laser pulses. The polarization state [linear (LIN), right circular (RC), and left circular (LC)] of the pump beam is controlled via a linear polarizer and a subsequent quarter-wave plate. (b) The measurement cycle for a discrimination experiment consists of one accumulation period for each of the three polarizations. a circling through the poles of the Poincare sphere, hence every ellipticity is achieved. The intensity after the analyzer is thus modulated with a sine function since only a one-dimensional projection of the polarization state can pass through the analyzer. Hence, the photodiode after the analyzer detects a sinusoidal signal with the same frequency as the EOM driving voltage. An optically active sample adds a rotation of the polarization plane, which translates to a change in the phase of the detected sinusoidal signal. By analyzing the photodiode signal with a lock-in amplifier we are able to detect OR changes of $0.10 \mathrm{mdeg}$ at an optical path length of only $250 \mu \mathrm{m} .{ }^{32}$ Note that we only detect OR changes $\Delta \alpha$, for absolute measurements a reference beam path would be necessary.

The CW probe is spatially overlapped with a fs pump beam (267 nm, $100 \mathrm{fs}, 1 \mathrm{kHz}$ ) in the sample capillary (Fig. 1a). The $30 \mu \mathrm{m}$ probe-beam diameter is small compared to the $200 \mu \mathrm{m}$ pumpbeam diameter. The fs pump pulses can be blocked with a mechanical shutter and their polarization state can be varied via a combination of a linear polarizer and a quarter-wave plate between linear (LIN), right circular (RC), and left circular (LC) polarization. Several subsequent fs laser pulses irradiate the same sample volume and every single pulse initiates a photoreaction. Even if the quantum efficiency for such a single photoreaction might be small, due to the accumulation scheme over many fs pulses the resulting signal is large enough to be detected.

For each pump-pulse polarization the following measurement procedure (Fig. 1b) is conducted five times: the sample in the capillary is renewed by moving the stepper motor. Then, the acquisition of the OR is started while the shutter is still closed providing a baseline for any light-induced OR changes $\Delta \alpha$. The averaged value of this data is subtracted from the complete data since we are only interested in $\Delta \alpha$. After $1 \mathrm{~s}$ the pump shutter is opened at $t=0$ and the sample is irradiated with UV laser pulses for $6 \mathrm{~s}$, while $\Delta \alpha$ is continuously recorded.

\section{Results and discussion}

We apply our method to 1,1'-binaphthyl-2,2'-diyl hydrogenphosphate (Sigma-Aldrich, CAS numbers: racemate 35193-63-6, $R$-enantiomer 39648-67-4, S-enantiomer 35193-64-7). Fig. 2 shows the CD spectra of the pure enantiomers and the racemate dissolved in methanol, as well as the absorption spectrum. While the racemate's CD spectrum is zero, the enantiomer spectra mirror each other. At the probe wavelength of $405 \mathrm{~nm}$, neither enantiomer absorbs, hence the probe does not excite any molecules but solely monitors OR.

To demonstrate the capabilities of our approach we first perform experiments with the pure enantiomers (either $R$ or $S$ ) in solution. The result of an accumulative OR experiment with the $S$-enantiomer in methanol (concentration $\gamma=13.5 \mathrm{mg} \mathrm{ml}^{-1}$ ) is shown in Fig. 3 (black solid line). Here, LIN polarization was utilized to trigger the photodecomposition. ${ }^{46}$ The result for the $R$-enantiomer (not shown) irradiated with LIN polarization is mirrored vertically at $\Delta \alpha=0$. For LC or RC the absolute 


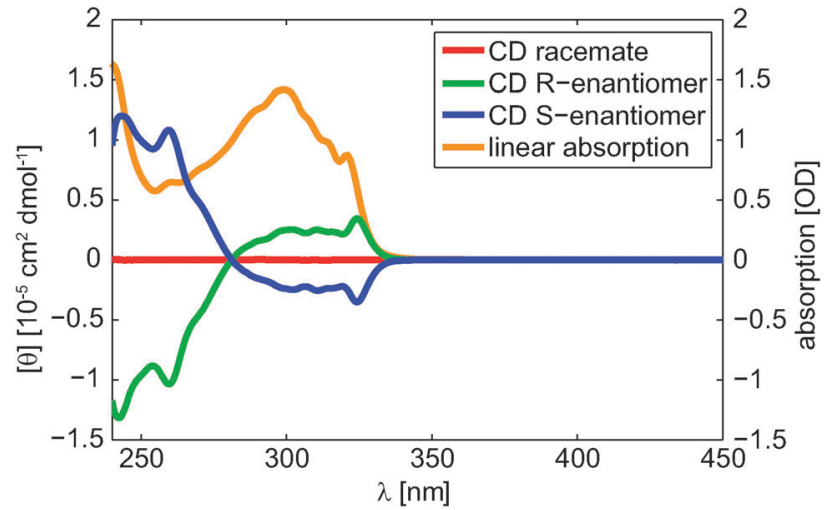

Fig. 2 Absorption (orange) and steady-state CD spectra in methanol of racemic $1,1^{\prime}$-binaphthyl-2,2' -diyl hydrogenphosphate (red), its pure $R$ - (green) and S-enantiomer (blue) at a concentration of $\gamma=0.5 \mathrm{mg} \mathrm{ml}^{-1}$. The Cotton effect for the electronic transition at $280 \mathrm{~nm}$ is clearly visible. Hence, by exciting the molecule with $267 \mathrm{~nm}$ we provide excess energy to the first excited state of the molecular system, making a photodecomposition more likely. Absorption is negligible at the OR probe wavelength of $405 \mathrm{~nm}$.

magnitude of the signal in Fig. 3 changes slightly, due to the slightly varying absorption of circular polarized light. However, as will be shown later, this effect is several orders of magnitude smaller than the measured OR change in Fig. 3. The exposure period is marked by the two black solid vertical lines in Fig. 3 but also before (waiting period) and after the exposure (diffusion period) OR data is recorded. Thus, for $t<0$ no pump pulses hit the sample, yielding a constant reference signal. This is used to be able to calculate the OR change $\Delta \alpha$. After opening the shutter at $t=0$, the signal changes until the pump is blocked again. The signal decay towards $\Delta \alpha=0$ afterwards is due to intact molecules diffusing into the probe volume. The complete curve of Fig. 3 can be described by modeling the photoreaction and including diffusion ${ }^{30,32}$ via

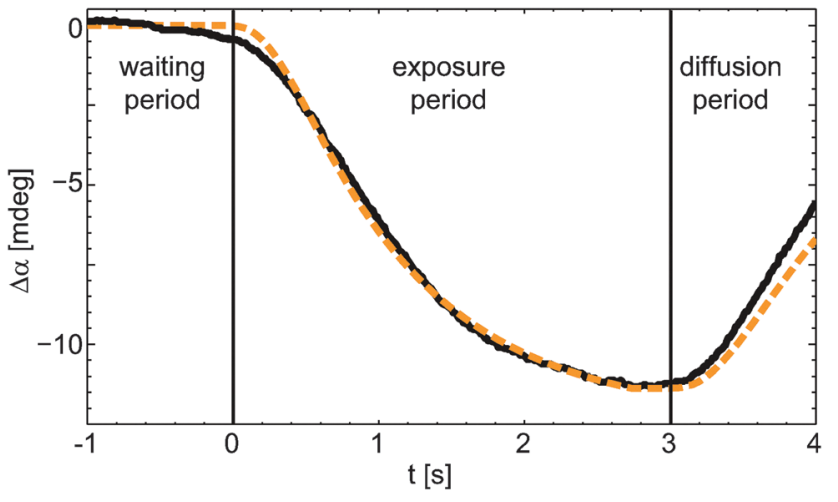

Fig. 3 Accumulative OR experiment (solid) and fit (dashed) for the S-enantiomer under LIN illumination with a pulse energy of $3.5 \mu \mathrm{J}$.

sub-volumes exhibit a different size (due to the different beam spot size of pump and probe beam) diffusion between these volumes and the surrounding volume can occur. ${ }^{30,32}$ This effect is included by the diffusion rate $d_{\mathrm{pu}}$ describing the exchange between pump volume and the surrounding volume and $d_{\mathrm{pr}}$ which refers to the diffusion between pump and probe volume. By convolving eqn (1) with the lock-in amplifier response function ${ }^{32}$

$$
\begin{aligned}
R_{\mathrm{LIA}}(t)= & \frac{2}{\sqrt{2 \mathrm{~s} \pi}(0.0805 \mathrm{~s}+0.2776 \mathrm{~s})} \\
& \times\left\{\Theta(0.2480 \mathrm{~s}-t) \exp \left[-\frac{(t-0.2480 \mathrm{~s})^{2}}{2(0.0805 \mathrm{~s})^{2}}\right]\right. \\
& \left.+\Theta(t-0.2480 \mathrm{~s}) \exp \left[-\frac{(t-0.2480 \mathrm{~s})^{2}}{2(0.2776 \mathrm{~s})^{2}}\right]\right\}
\end{aligned}
$$

where $\Theta(t)$ describes the Heaviside function, one can model the data in Fig. 3. The fit (dashed orange line in Fig. 3) reveals a

$$
\alpha_{\mathrm{pr}}(t, \tau)=\alpha_{0} \begin{cases}\frac{d_{\mathrm{pu}}+\eta \exp \left[-\left(d_{\mathrm{pu}}+\eta\right) t\right]}{d_{\mathrm{pu}}+\eta} & t<0 \\ \frac{d_{\mathrm{pu}} \exp \left[d_{\mathrm{pr}}(\tau-t)-\left(d_{\mathrm{pu}}+\eta\right) \tau\right]\left\{-1+\exp \left[\left(d_{\mathrm{pu}}+\eta\right) \tau\right]\right\} \eta}{\left(d_{\mathrm{pr}}-d_{\mathrm{pu}}\right)\left(d_{\mathrm{pu}}+\eta\right)} & 0 \leq t \leq \tau \\ +\frac{d_{\mathrm{pr}}\left(d_{\mathrm{pu}}+\left\{1-\exp \left[d_{\mathrm{pu}}(\tau-t)\right]+\exp \left(-d_{\mathrm{pu}} t-\eta \tau\right)\right\} \eta\right)}{\left(d_{\mathrm{pr}}-d_{\mathrm{pu}}\right)\left(d_{\mathrm{pu}}+\eta\right)} & \\ -\frac{d_{\mathrm{pu}}\left(d_{\mathrm{pu}}+\eta\right)}{\left(d_{\mathrm{pr}}-d_{\mathrm{pu}}\right)\left(d_{\mathrm{pu}}+\eta\right)} & t>\tau\end{cases}
$$

Here, $\alpha_{0}$ refers to the OR of the utilized molecular system at the probe wavelength of $405 \mathrm{~nm}$ and an optical path length of $250 \mu \mathrm{m}$, as used in the experiment. The $\mathrm{OR} \alpha_{\mathrm{pr}}(t, \tau)$ is present in the region of the capillary were the $\mathrm{CW}$ probe hits the sample before, during, and after the irradiation with fs pump pulses in the time period $\tau$, leading to photodecomposition with the rate $\eta$. Since these two maximum possible OR of $\alpha_{0}=19.18 \mathrm{mdeg}$ and diffusion constants $d_{\mathrm{pu}}=0.0643 \mathrm{~s}^{-1}$ and $d_{\mathrm{pr}}=2.9775 \mathrm{~s}^{-1}$, obtained as fit parameters.

As demonstrated above and previously for another molecular system,${ }^{32}$ it is possible to detect photoinduced OR changes in enantiopure samples. However, the goal of the presented work 


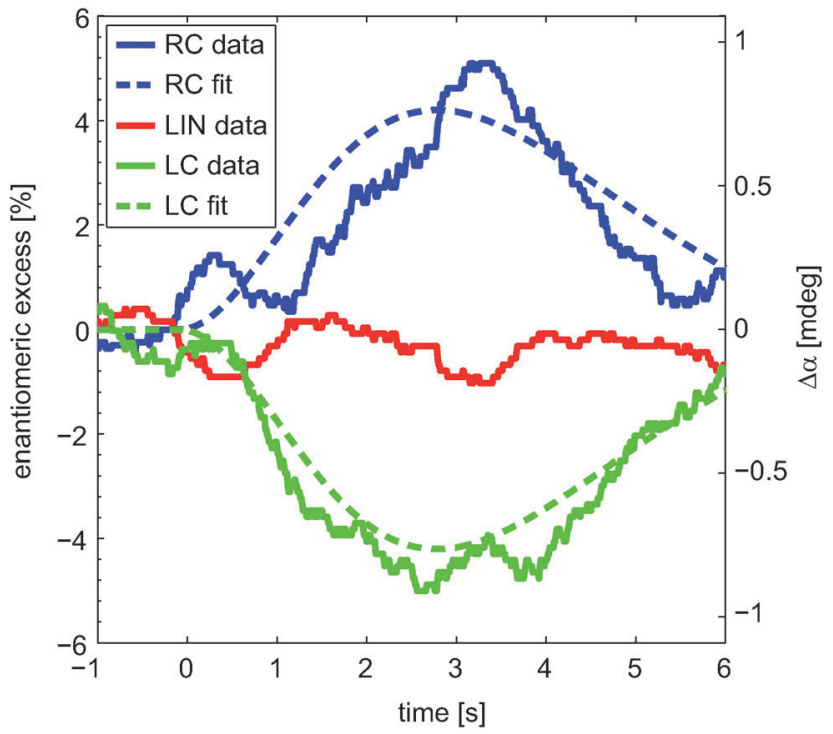

Fig. 4 Experimental outcome of an accumulative OR experiment for the racemate. While LIN pulses (red) do not change OR, LC pulses (green) decrease and RC pulses (blue) increase OR ( $\Delta \alpha$, right scale). For all three polarizations the pulse energy was $3.5 \mu \mathrm{J}$. Simulated curves [eqn (4), dashed lines] describe the data well within experimental uncertainties. The OR values can be mapped to ee values (left scale).

is to discriminate between achiral and racemic solutions, and hence an OR change in a racemate must be generated. Thus, we perform accumulative OR measurements using the scheme of Fig. $1 \mathrm{~b}$ with racemic solution $\left(\gamma=13.5 \mathrm{mg} \mathrm{ml}^{-1}\right)$. The results are shown in Fig. 4. For LIN pulses (red), no change in OR (right axis) is visible apart from noise. In contrast, RC pulses (blue) lead to a positive change, LC pulses (green) to a negative change. For RC and LC an extremum after $\approx 3 \mathrm{~s}$ of illumination is visible. Under UV irradiation the molecules form non-chiral fragments, ${ }^{46}$ but depending on the handedness of the polarization, more $R$ - or $S$-enantiomers are gradually decomposed, leading to an ee. Due to the small difference of the absorption coefficients of both enantiomers for LC or RC polarization, one fs pulse would not lead to a detectable signal. However, by accumulating the outcome of subsequent photoreactions, this photoinduced ee can be exposed throughout the measurement of Fig. 4.

In contrast to the case of Fig. 3 the pump irradiation is not stopped. Hence, the loss of signal magnitude for the OR change with RC and LC for $t>3 \mathrm{~s}$ must have a different origin. In the case of Fig. 4 the difference in the number of $R$ - and $S$-enantiomer molecules in the probed volume is increasing up to $t \approx 3 \mathrm{~s}$, hence the OR change is also increasing. However, due to the continuous irradiation with fs laser pulses the number of molecules are decreased. At one point all molecules in the probe volume are destroyed and only small contributions from intact molecules diffusing into the pumped volume are possible. In that case no OR change is detected, since a racemic sample as well as no sample molecules deliver the same OR value. Hence, the signal for circular polarizations in Fig. 4 must decrease again if the irradiation with fs pulses lasts long enough. As mentioned in the introduction such a behavior is already known ${ }^{34-36}$ and thus also theoretical approaches for kinetic modeling of ee are existent. In contrast to those $\mathrm{e}^{35,47,48}$ we describe the change in the number of molecules of the $R$-enantiomer upon RC illumination with first-order kinetics and combine that rate equation with diffusion into or out of the sample volume,

$$
\frac{\mathrm{d} N^{R}(t)}{\mathrm{d} t}=d_{\mathrm{pu}}\left(N_{0}^{R}-N^{R}(t)\right)-\Phi \sigma_{\mathrm{RC}}^{R} N^{R}(t) .
$$

Here, $N^{R}(t)$ denotes the number of molecules of the $R$-enantiomer at time $t$ and $N_{0}^{R}$ at $t=0, \Phi$ the photon flux impinging on the sample, and $d_{\mathrm{pu}}$ an effective diffusion constant that takes into account the irradiation geometry, ${ }^{30-32}$ like in eqn (1). Thus, since the beam alignment is not changed the determined value for $d_{\mathrm{pu}}$ from the experiment with the enantiopure solution (confer Fig. 3) is used in the following as a constant. The second diffusion constant $d_{\mathrm{pr}}$ is omitted since the irradiation is not stopped. We define $\sigma_{\mathrm{RC}}^{R}$ as the dimensionless cross section of the $R$-enantiomer for $\mathrm{RC}$ polarized light. It reflects the product of the molecular absorption coefficient and the quantum yield for decomposition in terms of a probability interpretation and is thus dimensionless, as e.g. also utilized in ref. 49. Hence, also the photon flux $\Phi$ is given in photons per second and lacks the reference to unit area in this case. It is also possible to use the conventional definition which however would lead to a further parameter which must be determined by the fitting routine. Since both parameters occur always as a product in the fitting formulas this is not meaningful, hence we chose the above mentioned definition. Each enantiomer has a slightly different cross section for the two polarizations, i.e., $\sigma_{\mathrm{RC}}^{R} \neq \sigma_{\mathrm{LC}}^{R}$ and $\sigma_{\mathrm{RC}}^{S} \neq \sigma_{\mathrm{LC}}^{S}$, while the relations $\sigma_{\mathrm{RC}}^{R}=\sigma_{\mathrm{LC}}^{S}$ and $\sigma_{\mathrm{LC}}^{R}=\sigma_{\mathrm{RC}}^{S}$ hold due to symmetry. Solving eqn (3) and its $S$-enantiomer analogue for the difference of the number of enantiomers upon RC illumination, $\Delta N(t)=N^{R}(t)-N^{S}(t)$, one obtains

$$
\begin{aligned}
& \Delta N(t)= \\
& N_{0}\left[\frac{d_{\mathrm{pu}}+\Phi \sigma_{\mathrm{RC}}^{R} \mathrm{e}^{-t\left(d_{\mathrm{pu}}+\Phi \sigma_{\mathrm{RC}}^{R}\right)}}{d_{\mathrm{pu}}+\Phi \sigma_{\mathrm{RC}}^{R}}-\frac{\left.d_{\mathrm{pu}}+\Phi \sigma_{\mathrm{RC}}^{S} \mathrm{e}^{-t\left(d_{\mathrm{pu}}+\Phi \sigma_{\mathrm{RC}}^{S}\right.}\right)}{d_{\mathrm{pu}}+\Phi \sigma_{\mathrm{RC}}^{S}}\right] \Theta(t)
\end{aligned}
$$

where $N_{0}=N_{0}^{R}=N_{0}^{S}$ because we have a racemate at and before $t=0$. The Heaviside function $\Theta(t)$ is needed since for $t<0$ no pump irradiation takes place. Eqn (4) changes sign if either the enantiomers $(R \leftrightarrow S)$ or the polarizations (LC $\leftrightarrow$ $\mathrm{RC})$ are exchanged. Similar to the enantiopure experiment, the convolution of eqn (4) with the response function of the lock-in amplifier, eqn (2), delivers fit curves as presented in Fig. 4 (dashed). From the fit parameters we retrieve the relative difference of the cross sections for $\mathrm{LC}$ and $\mathrm{RC}$ to be $\frac{\sigma_{\mathrm{RC}}^{R}-\sigma_{\mathrm{LC}}^{R}}{\frac{1}{2}\left(\sigma_{\mathrm{RC}}^{R}+\sigma_{\mathrm{LC}}^{R}\right)}=0.15 \%$. Even though the difference is small, our sensitive accumulative scheme enables following the kinetic evolution of the ee.

Since ee scales linearly with $\mathrm{OR}^{47,48}$ we calculate ee according to

$$
\mathrm{ee}(t)=\frac{N^{R}(t)-N^{S}(t)}{N_{0}^{R}+N_{0}^{S}}=\frac{\Delta \alpha(t)}{\alpha_{0}},
$$


with $\alpha_{0}$ determined from the fit of Fig. 3 . The right equality sign in eqn (5) holds only if no diffusion would be present in an experiment like the one presented in Fig. 4 since the "true" OR is diminished by diffusion. However, given the experimental noise it is a good approximation. This allows us to quantitatively obtain the ee axis in Fig. 4 (left). The plot reveals an ee of more than $4 \%$ at the optimal time point $t \approx 3 \mathrm{~s}$. If the irradiation is stopped at that point, this ee persists in the sample and only decreases via diffusion to and from the non-irradiated sample volume. The definition of the ee in eqn (5) describes the difference in the number of enantiomers present at time $t$ of the experiment divided by the initial total number of molecules. Typically, like in ref. 25, 47 and 48 also the denominator is chosen to be time-dependent, which leads to a diverging ee in the case when all molecules in the sample solution are destroyed. Hence, we chose to normalize to the initial total number of molecules [confer eqn (5)].

\section{Optical discrimination}

We now proceed to discuss how the detection of photoinduced ee, as presented in Fig. 4, can be used to discriminate racemic from achiral solutions. We hereby assume that we have dissolved one unknown molecular species in a known solvent. The linear absorption spectrum of this solution is known. Furthermore, also a static CD or OA spectrum is measured of the unknown solute but does not exhibit any signal. Hence, we know spectral regions where the unknown molecule absorbs, but we do not know if it is a racemic mixture or if the molecules in the solution are just achiral. We consider nine scenarios which might in general apply for the case described above. For all scenarios it is presumed that irradiation with fs pulses leads to a photoproduct which is different from the corresponding reactant, i.e., a photochemical reaction.

In this section we discuss optical discrimination of racemic from achiral solutions if the molecules under investigation interact with the fs pump pulses in the linear power regime. This is the simplest case, and can be verified with suitable attenuator series. For the situation of higher-order pump-power dependence see ESI. $\dagger$ For the analysis, we divided the above mentioned nine scenarios in four possible scenarios where only LIN pulses are used to irradiate the sample, as depicted in Fig. 5a and five possible scenarios in the case of circularly polarized pump pulses, presented in Fig. 5b. For LIN polarization our polarimeter will never measure a signal different from a flat line since no ee can be generated with LIN pulses.

The situation changes if one considers circularly polarized (LC or RC) pump pulses in scenarios 5-9, presented in Fig. 5b. Starting with an achiral molecular system in solution a photoreaction triggered by the absorption of one photon of the circularly polarized fs pulses always leads to no optical rotation change (see scenarios 5 and 6 in Fig. 5b). However, in the case of a racemic mixture as reactant the irradiation with fs laser pulses always leads to a non-zero optical rotation change (scenarios 7-9 in Fig. 5b). The data presented in Fig. 4 (LC and RC) corresponds (a)

linear polarization
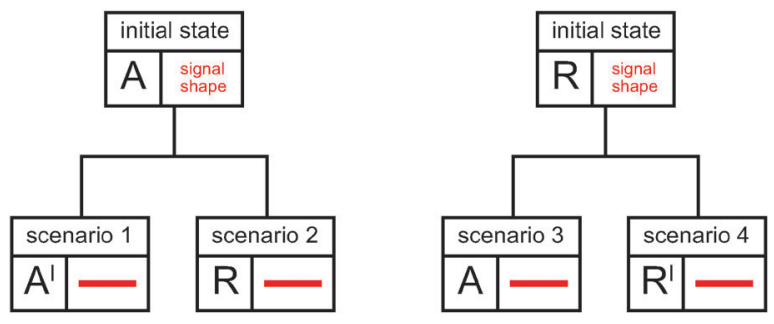

(b)

circular polarizations
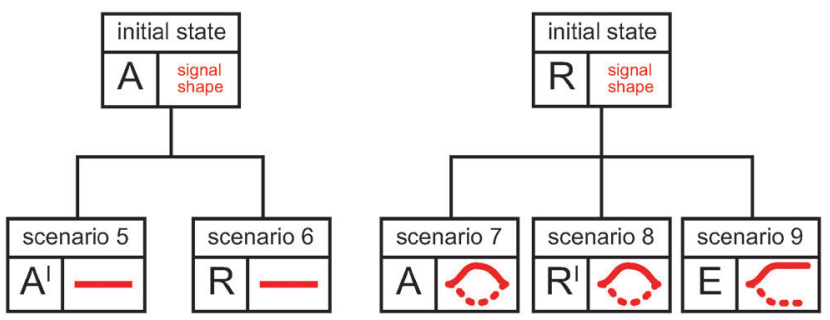

Fig. 5 Schematic representation of the measured optical rotation change after one interaction of the unknown solution with (a) linearly (LIN) or (b) circularly polarized (LC or RC) fs pump pulses. The signal shape is sketched in red (solid and dashed lines refer to the opposite handedness of the circular pump polarizations) for every scenario. Here, A refers to achiral molecules which differ from the achiral molecules $\mathrm{A}^{\prime}$. Analogously $R$ refers to a racemic mixture which differs from the racemate $R^{\prime}$. An enantiomeric excess is referred to with the letter $E$. (a) If the reactant in its initial state is achiral (left graph) or racemic (right graph), only an achiral or racemic solution can be generated photochemically with LIN pump pulses. Hence, in scenario 1-4 no optical rotation change can be observed and the signal corresponds to a flat line. (b) For initially achiral solutions also no optical rotation changes can be detected at any time with our approach if circular polarizations (LC or RC) are utilized. This changes if the sample under investigation is racemic. Here, either an achiral solution is generated (scenario 7), a different racemic mixture (scenario 8), i.e., the product molecules differ from the reactant molecules but still occur as a racemic mixture, or a persisting enantiomeric excess (scenario 9) is generated. In these three scenarios an optical rotation change can be detected. Thus, for one-photon interaction with the fs pump pulses optical discrimination is achievable by using circularly polarized fs pump pulses. If no optical rotation change is observed the sample is achiral; otherwise, if a signal is measured, the sample was initially racemic.

to scenario 7 in Fig. 5b. The shape of the curve would be similar if chiral, as racemic mixture, rather than achiral photoproducts were generated (scenario 8). For the special case that after a one-photon excitation with every fs laser pulse of the pulse train an ee could be generated, a constant offset signal for long illumination times would be measured (scenario 9).

Summarizing the results from all the various scenarios, optical discrimination of achiral and racemic solutions is straightforward with our approach in the linear power regime of pump-pulse interactions since the polarimeter never measures a signal if an achiral solution is the reactant (Fig. 5b, left graph) while a non-zero optical rotation change is observed in the case of a racemic mixture (Fig. 5b, right graph). The latter case was experimentally demonstrated in Fig. 4. Prolonged fs irradiation might lead to further photoreactions (see ESI $\dagger$ ) but 
as long as the chiral state of the product obtained with one photon is not changed this does not influence the discrimination capabilities of the presented method. Thus, optical discrimination between racemic and achiral solutions can be achieved if an excitation in the linear power regime causes a photoreaction for which the chirality properties of reactant and product differ.

\section{Outlook}

Summarizing, we have disclosed optical discrimination between various scenarios of achiral and racemic molecular solutions in the case of a linear dependence on the fs pump-pulse power. In particular, and in contrast to state-of-the-art methods, ${ }^{22-27}$ spatial separation is not required to discriminate racemic from achiral samples. Moreover, if the accumulative pump illumination is stopped at the right moment, the induced ee in the sample volume can be exploited for other purposes. Since the setup allows for fs time resolution ${ }^{32}$ future studies may investigate the ultrafast dynamics of chiral-molecule photoreactions. Beyond chiral discrimination, the presented setup can also be utilized for quantum control approaches ${ }^{50}$ which necessitate several interactions with fs pump pulses as theoretically proposed..$^{40,43,44}$ Here, the ambiguities derived in the ESI $\dagger$ are not of concern since in this case one typically knows the initial chirality state of the sample, but wants, e.g., to purify a racemic sample and thus enhance the achievable ee. For such purposes, the presented polarimeter can deliver a suitable feedback signal for closed- or open-loop quantum control approaches due to the demonstrated rapid enantioselective detection.

\section{Acknowledgements}

We thank Franziska Witterauf for the static CD measurements. PN acknowledges support within the Emmy-Noether program of the DFG and the Cluster of Excellence RESOLV (EXC 1069). AS thanks the Studienstiftung des deutschen Volkes for a scholarship.

\section{References}

1 D. B. Amabilino, Chirality at the nanoscale, Wiley-VCH, Weinheim, 2009.

2 G. Snatzke, Angew. Chem., Int. Ed., 1968, 7, 14-25.

3 J. P. Riehl and F. S. Richardson, Chem. Rev., 1986, 86, 1-16.

4 J. Meyer-Ilse, D. Akimov and B. Dietzek, Laser Photonics Rev., 2013, 7, 495-505.

5 P. Fischer and F. Hache, Chirality, 2005, 17, 421-437.

6 T. Dartigalongue and F. Hache, Chem. Phys. Lett., 2005, 415, 313-316.

7 C. Niezborala and F. Hache, J. Opt. Soc. Am. B, 2006, 23, 2418-2424.

8 L. Mangot, G. Taupier, M. Romeo, A. Boeglin, O. Cregut and K. D. H. Dorkenoo, Opt. Lett., 2010, 35, 381-383.

9 I. Eom, S. Ahn, H. Rhee and M. Cho, Opt. Express, 2011, 19, 10017-10028.
10 M. Bonmarin and J. Helbing, Ultrafast Phenomena XVII, 2011, pp. 862-864.

11 J. Helbing and M. Bonmarin, J. Chem. Phys., 2009, 131, 174507.

12 M. Bonmarin and J. Helbing, Chirality, 2009, 21, E298-E306.

13 X. Xie and J. D. Simon, Biochemistry, 1991, 30, 3682-3692.

14 S. Milder, S. Bjorling, I. Kuntz and D. Kliger, Biophys. J., 1988, 53, 659-664.

15 J. W. Lewis, R. A. Goldbeck, D. S. Kliger, X. Xie, R. C. Dunn and J. D. Simon, J. Phys. Chem., 1992, 96, 5243-5254.

16 A. Trifonov, I. Buchvarov, A. Lohr, F. Würthner and T. Fiebig, Rev. Sci. Instrum., 2010, 81, 043104.

17 J. Meyer-Ilse, D. Akimov and B. Dietzek, J. Phys. Chem. Lett., 2012, 3, 182-185.

18 C. Lux, M. Wollenhaupt, T. Bolze, Q. Liang, J. Köhler, C. Sarpe and T. Baumert, Angew. Chem., Int. Ed., 2012, 51, 5001-5005.

19 G. A. Garcia, L. Nahon, S. Daly and I. Powis, Nat. Commun., 2013, 4, 2132.

20 D. Patterson, M. Schnell and J. M. Doyle, Nature, 2013, 497, 475-477.

21 M. Pitzer, M. Kunitski, A. S. Johnson, T. Jahnke, H. Sann, F. Sturm, L. P. H. Schmidt, H. Schmidt-Böcking, R. Dörner, J. Stohner, J. Kiedrowski, M. Reggelin, S. Marquardt, A. Schieer, R. Berger and M. S. Schöffler, Science, 2013, 341, 1096-1100.

22 A. Eilam and M. Shapiro, Phys. Rev. Lett., 2013, 110, 213004.

23 A. Canaguier-Durand, J. A. Hutchison, C. Genet and T. W. Ebbesen, New J. Phys., 2013, 15, 123037.

24 R. P. Cameron, S. M. Barnett and A. M. Yao, New J. Phys., 2014, 16, 013020.

25 D. Parker, Chem. Rev., 1991, 91, 1441-1457.

26 T. E. Beesley and R. P. W. Scott, Chiral chromatography, Wiley, Chichester, 1998.

27 H. Lorenz and A. Seidel-Morgenstern, Angew. Chem., Int. Ed., 2014, 53, 1218-1250.

28 M. Quack, Angew. Chem., Int. Ed., 2002, 41, 4618-4630.

29 R. Berger, G. Laubender, M. Quack, A. Sieben, J. Stohner and M. Willeke, Angew. Chem., Int. Ed., 2005, 44, 3623-3626.

30 F. Langhojer, F. Dimler, G. Jung and T. Brixner, Opt. Lett., 2007, 32, 3346-3348.

31 F. Langhojer, F. Dimler, G. Jung and T. Brixner, Biophys. J., 2009, 96, 2763-2770.

32 A. Steinbacher, J. Buback, P. Nuernberger and T. Brixner, Opt. Express, 2012, 20, 11838-11854.

33 Y. Inoue and V. Ramamurthy, Chiral Photochemistry, CRC Press, 2004.

34 W. Kuhn and E. Braun, Naturwissenschaften, 1929, 17, 227-228.

35 W. Kuhn and E. Knopf, Z. Phys. Chem. B, 1930, 7, 292-310.

36 S. Mitchell, J. Chem. Soc., 1930, 1829-1834.

37 J. Shao and P. Hänggi, J. Chem. Phys., 1997, 107, 9935-9941.

38 A. Salam and W. J. Meath, Chem. Phys., 1998, 228, 115-129.

39 Y. Fujimura, L. González, K. Hoki, J. Manz and Y. Ohtsuki, Chem. Phys. Lett., 1999, 306, 1-8. 
40 M. Shapiro, E. Frishman and P. Brumer, Phys. Rev. Lett., 2000, 84, 1669.

41 P. Brumer, E. Frishman and M. Shapiro, Phys. Rev. A: At., Mol., Opt. Phys., 2001, 65, 015401.

42 S. S. Bychkov, B. A. Grishanin and V. N. Zadkov, J. Exp. Theor. Phys., 2001, 93, 24-32.

43 K. Hoki, L. González and Y. Fujimura, Chem. Phys., 2002, 116, 8799-8802.

44 D. Gerbasi, M. Shapiro and P. Brumer, J. Chem. Phys., 2006, 124, 074315.

45 D. V. Zhdanov and V. N. Zadkov, J. Chem. Phys., 2007, 127, 244312.
46 For the photochemical reaction pathway of a similar molecule see, M. Flegel, M. Lukeman and P. Wan, Can. J. Chem., 2008, 86, 161-169.

47 P. Newman, P. Rutkin and K. Mislow, J. Am. Chem. Soc., 1958, 80, 465-473.

48 G. Balavoine, A. Moradpour and H. B. Kagan, J. Am. Chem. Soc., 1974, 96, 5152-5158.

49 J. F. Widmann, J. C. Yang, T. J. Smith, S. L. Manzello and G. W. Mulholland, Combust. Flame, 2003, 134, 119-129.

50 P. Nuernberger, G. Vogt, T. Brixner and G. Gerber, Phys. Chem. Chem. Phys., 2007, 9, 2470-2497. 